\title{
Early Experience of the Use of Cyanoacrylate Glue in Superficial Venous Disease in an Asian Population: A Pilot Study
}

\author{
Kai Siang CHAN ${ }^{1,2}$, Zhiwen Joseph LO ${ }^{2, ~ *}$, Qiantai HONG ${ }^{2}$, Enming YONG ${ }^{2}$, Li ZHANG ${ }^{2}$, \\ Sadhana CHANDRASEKAR ${ }^{2}$, Glenn Wei Leong TAN ${ }^{2}$ \\ ${ }^{I} \mathrm{MOH}$ Holdings, Singapore \\ ${ }^{2}$ Vascular Surgery Service, Department of General Surgery, Tan Tock Seng Hospital, Singapore
}

*Corresponding Author: Zhiwen Joseph LO, Vascular Surgery Service, Department of General Surgery, Tan Tock Seng Hospital, Singapore

\begin{abstract}
Background: The use of cyanoacrylate glue has garnered attention in the treatment of chronic venous insufficiency as it poses no risk to surrounding structures. This is a feasibility study accessing the technical success and safety profile on the use of a new technique of using cyanoacrylate glue for superficial venous disease in an Asian population.
\end{abstract}

Methods: This is a retrospective review of a prospective database, conducted on patients treated between July and August 2019. Patient demographics, peri-operative and post-operative outcomes were recorded.

Results: A total of 11 great saphenous veins in 7 patients were treated. The median age was 59.8 with 3 males (42.9\%). The median operating time is $25 \mathrm{~min}$ and $50 \mathrm{~min}$ for the unilateral and bilateral subgroup respectively. Median cost of consumables expressed as a percentage of other treatment modalities are as follows: $109.5 \%$ of VNUS® radiofrequency ablation, $146.0 \%$ of EVRF® radiofrequency ablation, $41.7 \%$ of VenaSeal ${ }^{\mathrm{TM}}$ cyanoacrylate glue and $97.3 \%$ of ClariVein ${ }^{\circledR}$ Mechanochemical ablation. We report $100 \%$ occlusion rates immediately following the procedure with one caseof phlebitis and no deep vein thrombosis. There was no recurrence during the 6-months surveillance. There was an significant improvement in VCSS post-operatively (median 3 vs 12, p=0.017). EQ-5D also improved post-operatively, though non-significant (median 80 vs 70, $p=0.461$ ).

Conclusion: Use of cyanoacrylate glue to treat superficial venous disease in an Asian population is safe and feasible.

Keywords: superficial venous disease; chronic venous disease; chronic venous insufficiency; cyanoacrylate glue; cyanoacrylate adhesives.

\section{INTRODUCTION}

Chronic venous insufficiency (CVI) is a common disease with an incidence of up to $50 \%$ worldwide with varying incidences regionally. ${ }^{1,2}$ Mild to moderate CVI may manifest itself as itch, burning sensation or pain with significant impact on quality of life. ${ }^{3}$ Venous leg ulcer is a more severe complication of CVI with significant socioeconomic burden. ${ }^{4}$

Management of CVI has seen a vast transformation from surgical stripping to sclerotherapy, endovenous laser therapy (EVLT), radiofrequency ablation (RFA) and mechanical occlusion chemically-assisted ablation (MOCA).Studies have demonstrated these minimally invasive methods are noninferior and at least as effective as surgery in the treatment of varicose veins, but with fewer complications and less post-operative pain., ${ }^{5,8}$ However, thermal ablation with laser or radiofrequency poses a risk of thermal injury to surrounding structures and requiresthe administration of tumescent anaesthesiawhich prolongs operation time. ${ }^{9}$

Recently, the use of cyanoacrylate glue has garnered attention in the management of CVI and treatment of the great saphenous vein (GSV). Cyanoacrylate glue are non-thermal and non-tumescent, and hence, poses no risk of injury to surrounding structures. Various techniques have been developed for the use of cyanoacrylate glue in treating superficial venous disease: theVenaSeal $^{\mathrm{TM}} \quad$ ClosureSystem (Medtronic, Minneapolis, Minnesota) and the 
Veinecto system(fCare Systems ${ }^{\circledR}$, Antwerp, Belgium). Feasibility studies have shown that the VenaSeal ${ }^{\mathrm{TM}}$ Closure System is comparable to thermal procedures in closure rates and is safe. ${ }^{10,11}$ However, there are no existing studies reporting the outcomes of the Veinecto system in an Asian population thus far. Hence, this study aims to provide a pilot review on early experience with the use of cyanoacrylate glue (Veinecto system) on superficial venous disease in an Asian population.

\section{MATerials AND Methods}

This is a retrospective review of a prospective database, conducted on seven patients who were treated with cyanoacrylate glue using the Veinecto system for superficial venous disease between July and August 2019 at a 1700-bed tertiary university-affiliated teaching hospitalin Singapore. Although the Singapore Health Sciences Authority approved the usage of Veinecto cyanoacrylate glue system, it was a new product to the local healthcare system and hencewe elected to perform a pilot study and evaluate the 6-month outcomes, prior to complete adoption for all patients.

\subsection{Patient Selection}

Inclusion criteria were patients with superficial venous disease classified as $\mathrm{C} 3$ to $\mathrm{C} 6$ of the Clinical, Etiology, Anatomy, Pathophysiology (CEAP) classification system. ${ }^{12}$ Exclusion criteria were patients who were pregnant, documented hypersensitivity to cyanoacrylatebased products or high-density liquid embolisation agents and history of thrombophlebitis or had sepsis within the last month were excluded from the treatment using the Veinecto system as per the manufacturer's instructions.All patients with the abovementioned criteria were offered EVLT, RFA, MOCA and cyanoacrylate glue as treatment modalities for superficial venous disease. Decision for cyanoacrylate glue was based on shared decision making between the surgeon and patient. All patients were identified electronically using the hospital electronic records and operative database.Data was extracted from the Chronic Venous Disease Registry, a standing database approved by the local institutional review board (National Healthcare Group Domain Specific Review Board, reference no: TTSH/2018-00039). This study has been exempted from ethical board approval by the local institutional review board (National Healthcare Group Domain Specific
Review Board, reference no: 2020/00314).Written informed consentwas obtained from allpatients included in this study on the use of collected data and non-identifiable intra-operative images for research publications, as part of the Chronic Venous Disease Registry.

\subsection{Study Variables, Outcome Measures and Definitions}

Study variables include patient demographics, quality of life (QoL) using the EQ-5D questionnaire, ${ }^{13}$ severity of venous disease,perioperative, post-operative details and costs of surgery and hospitalisation stay. The EQ-5D questionnaire is routinely administered in our institution for any patients presenting with superficial venous disease. Our main study outcomes are occlusion rates, recurrence and morbidity.Occlusion was defined as presence of thrombosis on the duplex ultrasound following cyanoacrylate glue administration. Recurrence was defined as patency of the treated vein detected on duplex ultrasound at 6-week followup.Morbiditywas defined as phlebitis, infection and deep vein thrombosis (DVT). Severity of venous disease was assessed using the CEAP classification and the Venous clinical severity score (VCSS). ${ }^{14}$ Operating time was calculated from initial vein puncture till sheath removal.Phlebitis was defined as erythema, pain or edema occurring over the area of the treated vein. Cost of consumables was defined as costs of device and disposables used in the surgery. Cost of consumables was expressed as a percentage of alternative modalities of treatment for chronic venous insufficiency commonly used in the market at present: VNUS ${ }^{\circledR}$ radiofrequency ablation (VNUS Medical Technologies, California, United States of America), EVRF® radiofrequency ablation (fCare Systems ${ }^{\circledR}, \quad$ Antwerp, Belgium), VenaSeal $^{\mathrm{TM}}$ cyanoacrylate glue and ClariVein ${ }^{\circledR}$ mechanochemical ablation (Merit Medical, Utah, United States of America). EQ-5D questionnaire and VCSS score were obtained pre-operatively (at specialist outpatient clinic 1 week prior to surgery) and on review 6-week post-operatively.

\subsection{Treatment Procedure}

All patients were given prophylactic antibiotics with a single dose of intravenous cefazolin perioperatively according to the local institution antibiogram guidelines. Patients were administered local or general anaesthesia as per required. Decision for local or general 
anaesthesia was based on shared decision making between the anaesthetist and patient.

A 6 French introducer sheath and peripheral braided microcatheter were inserted either in a retrograde or antegrade fashion (Figure 1). For retrograde approaches, the peripheral braided microcatheter was positioned $5 \mathrm{~cm}$ distal to the saphenofemoral junction (SFJ) under ultrasound guidance. The retrograde approaches were adopted for patients without SFJ or proximal GSV reflux. This approach allows for targeted treatment of GSV lengths with reflux and also enables treatment of distal GSV along the distal calf region. For antegrade approaches, the peripheral braided microcatheter was position as distally as possible under ultrasound guidance.
The antegrade approaches were adopted for patients with SFJ or proximal GSV reflux. The delivery catheter is then introduced and positioned with the Veinecto dispenser gun was attached to the delivery catheter for administering of the Veinectoembolisation agent. The catheter was then retracted slowly with administration of the Veinectoembolisation agent at $4 \mathrm{~cm}$ intervals.After complete withdrawal of the catheter, firm pressure was applied to the entry site for two minutes. Duplex ultrasound was used to confirm occlusion of the treated vein.

Figure 1: Introduction of peripheral braided microcatheter were inserted either in a retrograde or antegrade fashion

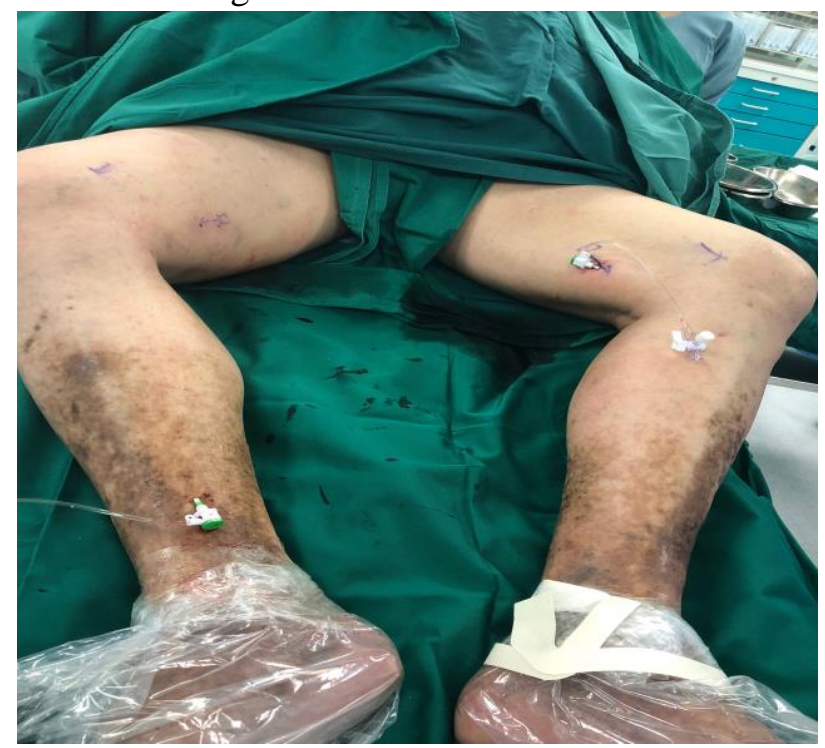

Patients were monitored in the ambulatory surgery monitoring ward overnight and were discharged the following morning if clinically well. Patients were instructed to ambulate frequently after the procedure and were able to return to work or resume daily activities as tolerated. Routine non-steroidal antiinflammatory agents (NSAIDs) were prescribed for 5 days for all patients.

All patients were routinely followed-up at the specialist outpatient clinic at 6-weeks and 6months postoperatively. The EQ-5D questionnaire, VCSS and duplex ultrasound were administered in the follow-up visit at week 6 postoperatively as part of routine institution monitoring for patients post-treatment for superficial venous disease. Duplex ultrasound was performed 6-months postoperatively for surveillance of recurrence.

\subsection{Statistical analysis}

All statistical analysis was performed using SPSS version 25.0 (SPSS Inc., Chicago, III., USA). All categorical variables were expressed as number (\%) and all continuous variables were expressed as median (interquartile range). Continuous variables were expressed as median due to the small population in this study. Wilcoxon-signed rank test was used to compare pre-operative versus post-operativeEQ-5D score and VCSS scores. A p-value $<0.05$ was deemed as statistically significant.

\section{Results}

A total of 11 GSVs in 7 patientswere treated with theVeinecto system during this study period. One patient had additional treatment for bilateral anterior accessory GSV (AAGSV). The median age was 59.8 (IQR 56.5-71.4) with 3 males $(42.9 \%)$. Majority of the patients $(n=3$, 42.9\%) had C6 of the CEAP classification. Three patients $(42.9 \%)$ had previous surgeries for varicose veins. Pre-operative median EQ-5D 
Early Experience of the Use of Cyanoacrylate Glue in Superficial Venous Disease in an Asian Population: A Pilot Study

and VCSS score was 70 and 12 respectively. is summarised in Table 1.

Baseline characteristics of the treated population

Table 1: Demographics and clinical profile of the treated population $(n=7)$

\begin{tabular}{|l|l|}
\hline & \multicolumn{1}{c|}{$\mathbf{n}(\%)$ or median (IQR) } \\
\hline Age & $59.8(56.5-71.4)$ \\
\hline Gender, male & $3(42.9)$ \\
\hline BMI, $\mathrm{kg} / \mathrm{m}^{2}$ & $32(29-38)$ \\
\hline ASA score $\geq 3$ & $4(57.1)$ \\
\hline CEAP classification & \\
Clinical & $1(14.3)$ \\
C3 & $2(28.6)$ \\
C4 & $1(14.3)$ \\
C5 & $3(42.9)$ \\
C6 & $7(100)$ \\
\hline Etiology, primary & \\
\hline Anatomy & $7(100)$ \\
Superficial & $2(28.6)$ \\
Deep & \\
\hline Side of operation & $3(42.9)$ \\
Unilateral & $4(57.1)$ \\
Bilateral & $3(42.9)$ \\
\hline Previous varicose vein surgery & $70(70-80)$ \\
\hline Pre-operative EQ-5D score & $12(9-13)$ \\
\hline Pre-operative VCSS &
\end{tabular}

All continuous variables are expressed as median (interquartile range) unless stated otherwise

ASA: American Society of Anaesthesiology, BMI: Body mass index; CEAP: Clinical, Etiology, Anatomy, Pathophysiology; VCSS: Venous Clinical Severity Score

Table 2 summarises the peri-operative characteristics of the treated population. The median overall operating time is $50 \mathrm{~min}$ (IQR 25-50), $25 \mathrm{~min}$ (IQR 25-35) for the unilateral subgroup and $50 \mathrm{~min}$ (IQR 50-53.8) for the bilateral subgroup. Median length of GSV

Table 2: Peri-operative details and procedure characteristics $(n=7)$

\begin{tabular}{|l|l|}
\hline & \multicolumn{1}{|c|}{ n (\%) or median (IQR) } \\
\hline Type of anaesthesia, n (\%) & $3(42.9)$ \\
Local & $4(57.1)$ \\
General & $50(25-50)$ \\
\hline Operating time, median (IQR), min & $25\left(25-35^{\wedge}\right)$ \\
Unilateral & $50(50-53.8)$ \\
Bilateral & $30(25-42)$ \\
\hline Length of all GSV, cm & $30(24-52)$ \\
Length of right GSV, cm & $33(25-43)$ \\
\hline Length of left GSV, cm & $25(25-25)$ \\
\hline Length of AAGSV, cm & $25^{*}$ \\
Length of right AAGSV, cm & $25^{*}$ \\
Length of left AAGSV, cm & \\
\hline Median cost of consumables expressed as a ratio of alternative & \\
modalities of treatment & $109.5 \%$ \\
VNUS Radiofrequency ablation $_{\text {EVRF }}$ Radiofrequency ablation & $146.0 \%$ \\
VenaSeal & \\
ClariVein & \\
\hline
\end{tabular}

All continuous variables are expressed as median (interquartile range) unless stated otherwise

"Median is unable to be calculated as there is only a single value treated was $30 \mathrm{~cm}$ (IQR $25-42 \mathrm{~cm})$. Length of AAGSV treated was $25 \mathrm{~cm}$. The median cost of consumables was expressed as a percentage of the cost of alternative treatment modalities for chronic venous insufficiency. 
Early Experience of the Use of Cyanoacrylate Glue in Superficial Venous Disease in an Asian Population: A Pilot Study

${ }^{1} 100^{\text {th }}$ quartile is presented as $75^{\text {th }}$ quartile is unavailable

Table 3 summarises the post-operative outcomes of the treated veins. Majority of the patients did not experience any post-operative complications. Phlebitis occurred in one patient with one vein $(9.1 \%)$, which resolved with a further week of NSAIDs and did not require any

Table 3: Post-operative outcomes of treated veins $(n=11)$

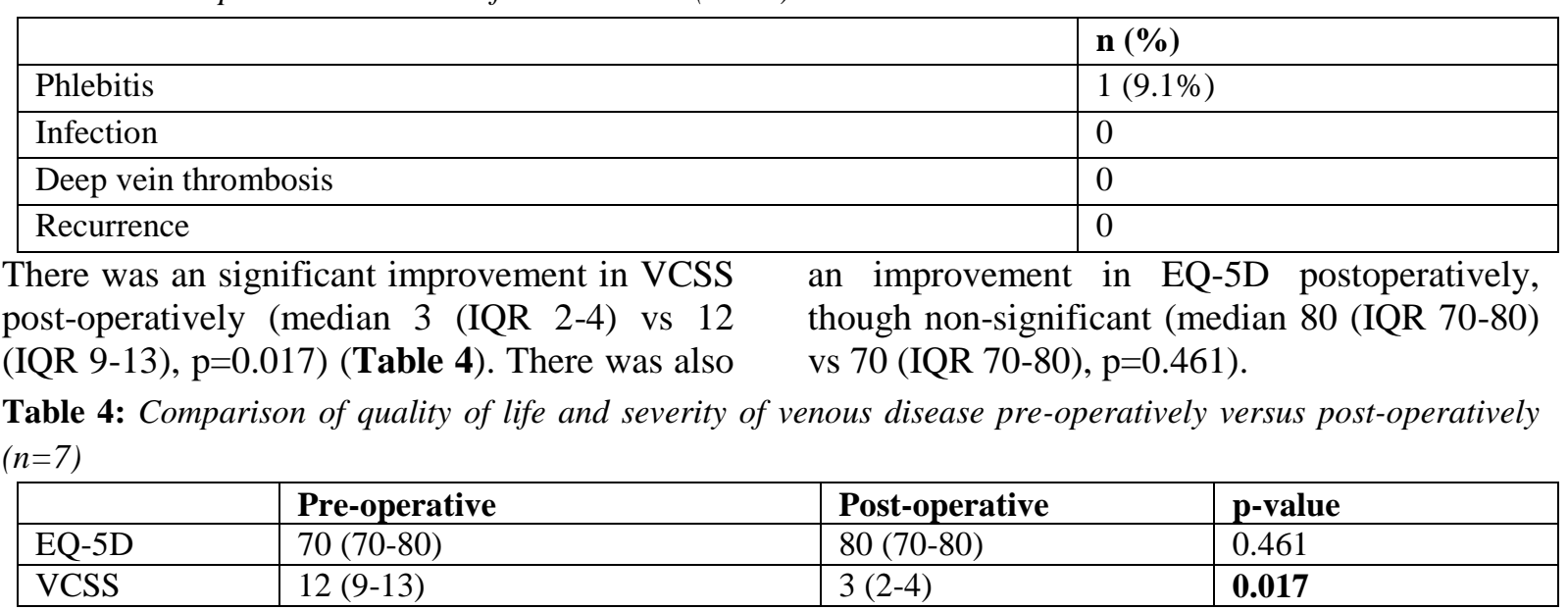

All continuous variables are expressed as median (interquartile range) unless stated otherwise

VCSS: Venous Clinical Severity Score

\section{Discussion}

The use of cyanoacrylate glue in treatment of superficial venous disease has a history of less than a decade..$^{15}$ In contrary to traditional minimally-invasive endovenous ablation techniques, cyanoacrylate glue are non-thermal and non-tumescent, posing no risk of thermal injury to surrounding structures and absolves the need for tumescent anaesthesia. Existing studies have reported the feasibility and efficacy of the VenaSeal $^{\mathrm{TM}}$ Closure System showing high closure rates and improvement in VCSS. ${ }^{11}$ This is however to our knowledge, the first study to report the use of the Veinecto system in the treatment of superficial venous disease.

Our study demonstrates feasibility of the use of cyanoacrylate glue with the Veinecto system for treatment of superficial venous disease.We obtained $100 \%$ closure rates at 6-month followup, though this was only conducted in a small sample size. The closure rates in our study are similar to existing studies on superficial venous disease treatment using the VenaSeal ${ }^{\mathrm{TM}}$ Closure System. A study by Park et al on 47 GSV and 16 SSV reported a $100 \%$ closure rate at 1-month follow-up (25 patients with 42 treated veins had follow-up), where closure was defined as less than $5 \mathrm{~cm}$ length of patency. ${ }^{10} \mathrm{~A}$ study by Alm et al similar reported high closure rates of $96.5 \%$ antibiotics. There was no incidence of DVT. Surveillance on ultrasound duplex revealed thrombosis of all the treated GSVs at 6weeks. Median length of follow up was 6 months with no recurrence. 
Our study also demonstrated the safety of the Veinecto system: there was one occurrence $(9.1 \%)$ of phlebitis. None of the treated cases had any DVT. The issue of DVT is of concern in cyanoacrylate use as the close proximity of the catheter to the SFJ may predispose extension of cyanoacrylate glue into the deep venous system. Studies have recommended a $4.0-5.0 \mathrm{~cm}$ distance from the SFJ to avoid the risk of cyanoacrylate glue extension into the deep venous system. ${ }^{15}$ In our study, the catheter was positioned $5 \mathrm{~cm}$ from the $\mathrm{SFJ}$ and we did not observe any DVT in the treated population. Nevertheless, the risk of DVT is definitely a concern and further studies should be carried out on the recommended distance of positioning of the catheter from the SFJ to reduce the risk of cyanoacrylate glue extension to the deep venous system.

Apart from the feasibility and safety profile of the use of the Veinecto system, our study demonstrated a non-significant improvement in quality of life (median EQ-5D score 80 vs 70 , $\mathrm{p}=0.461$ ). The lack of statistical significance is likely due to the small sample size of our study. QoL is an important outcome measure in the management in chronic venous disease, ${ }^{18}$ but has only been explored in a minority of the studies. Alm et al described better improvement in QoL compared to thermal procedures as patients were able to resume daily activities immediately after treatment without the need of compression therapy. ${ }^{11}$ However, there was no objective marker of QoL in their study. We also included the cost of consumables in the study referenced to other commonly used modalities of treatment in the market for chronic venous insufficiency. The use of cyanoacrylate glue is more costly than thermal ablation methods due to the need for more consumables as demonstrated in our study. Chan et al described the cost issues of cyanoacrylate glue and recommended its use for bilateral cases as a bottle of cyanoacrylate glue could be used to treat bilateral superficial venous disease. ${ }^{19}$ As studies continue to show the feasibility, efficacy and safety of the use of cyanoacrylate glue, cost-benefit analysis should be provided to justify its use over other treatment modalities.

This study has several limitations. Firstly, this is a retrospective study with a small sample size with inherent selection bias. However, this study is a pilot study to report our early experience with the use of the Veinecto system as no prior studies have reported its efficacy and safety profile in an Asian population. It has been described in literature that a minimum sample size of 10-12 for pilot studies provides the ability to test a hypothesis. ${ }^{20}$ Our study included 7 patients with 11 treated veins. We also did not assess the level of pain using the numerical rating scale or visual analogue scale postoperatively. However, pain was assessed in both the EQ-5D and the VCSS. Unfortunately, we are also unable to explicitly state the cost of the use of the Veinecto system in view of the sensitive nature of this information, but we have expressed the cost as a percentage of alternative modalities of management for ease of comparison for readers.

\section{CONCLUSION}

Within our pilot study on the use of cyanoacrylate glue (Veinecto system) in the treatment of superficial venous disease in an Asian population, there are good clinical outcomes with good safety profile and minimal complications.Further prospective studies should be carried out to validate these findings.

\section{REFERENCES}

[1] Brand FN, Dannenberg AL, Abbott RD, et al. The epidemiology of varicose veins: the Framingham Study. American journal of preventive medicine 1988; 4: 96-101.

[2] Zahariev T, Anastassov V, Girov K, et al. Prevalence of primary chronic venous disease: the Bulgarian experience. International angiology 2009; 28: 303.

[3] Duque MI, Yosipovitch G, Chan YH, et al. Itch, pain, and burning sensation are common symptoms in mild to moderate chronic venous insufficiency with an impact on quality of life. Journal of the American Academy of Dermatology 2005; 53: 503-507.

[4] Lo ZJ, Lim X, Eng D, et al. Clinical and economic burden of wound care in the tropics: a 5- year institutional population health review. International Wound Journal 2020.

[5] Biemans AA, Kockaert M, Akkersdijk GP, et al. Comparing endovenous laser ablation, foam sclerotherapy, and conventional surgery for great saphenous varicose veins. Journal of vascular surgery 2013; 58: 727-734. e721.

[6] Pan Y, Zhao J, Mei J, et al. Comparison of endovenous laser ablation and high ligation and stripping for varicose vein treatment: a metaanalysis. Phlebology 2014; 29: 109-119.

[7] Nesbitt C, Bedenis R, Bhattacharya V, et al. Endovenous ablation (radiofrequency and laser) and foam sclerotherapy versus open surgery for 
great saphenous vein varices. Cochrane Database of Systematic Reviews 2014.

[8] Darvall K, Bate G, Adam D, et al. Recovery after ultrasound- guided foam sclerotherapy compared with conventional surgery for varicose veins. British Journal of Surgery: Incorporating European Journal of Surgery and Swiss Surgery 2009; 96: 1262-1267.

[9] Korkmaz K, Yener AÜ, Gedik HS, et al. Tumescentlessendovenous radiofrequency ablation with local hypothermia and compression technique. Cardiovascular journal of Africa 2013; 24: 313.

[10] Park I. Initial outcomes of cyanoacrylate closure, VenaSeal system, for the treatment of the incompetent great and small saphenous veins. Vascular and endovascular surgery 2017; 51: 545-549.

[11] Alm J. VenaSeal ${ }^{\mathrm{TM}}$ closure treatment of saphenous varicosis. Phlebologie 2014; 43: 242-248.

[12] Eklöf B, Rutherford RB, Bergan JJ, et al. Revision of the CEAP classification for chronic venous disorders: consensus statement. Journal of vascular surgery 2004; 40: 1248-1252.

[13] Carradice D, Mazari F, Samuel N, et al. Modelling the effect of venous disease on quality of life. British journal of surgery 2011; 98: 1089-1098.
[14] Vasquez $M$ and Munschauer C. Venous Clinical Severity Score and quality-of-life assessment tools: application to vein practice. Phlebology 2008; 23: 259-275.

[15] Lam YL, De Maeseneer M, Lawson J, et al. Expert review on the VenaSeal ${ }^{\circledR}$ system for endovenouscyano-acrylate adhesive ablation of incompetent saphenous trunks in patients with varicose veins. Expert review of medical devices 2017; 14: 755-762.

[16] Lane TR, Kelleher D, Moore HM, et al. Cyanoacrylate glue for the treatment of great saphenous vein incompetence in the anticoagulated patient. Journal of Vascular Surgery: Venous and Lymphatic Disorders 2013; 1: 298-300.

[17] Proebstle T, Alm J, Dimitri S, et al. Twelvemonth follow-up of the European multicenter study on cyanoacrylate embolization of incompetent great saphenous veins. Journal of Vascular Surgery: Venous and Lymphatic Disorders 2014; 2: 105-106.

[18] van Korlaar I, Vossen C, Rosendaal F, et al. Quality of life in venous disease. Thrombosis and haemostasis 2003; 90: 27-35.

[19] Chan YC, Law Y, Cheung GC, et al. Cyanoacrylate glue used to treat great saphenous reflux: measures of outcome. Phlebology 2017; 32: 99-106.

[20] Van Belle G. Statistical rules of thumb. John Wiley \& Sons, 2011.

Citation: Kai Siang CHAN, Zhiwen Joseph LO, Qiantai HONG, Enming YONG, Li ZHANG, Sadhana CHANDRASEKAR, Glenn Wei Leong TAN. Early Experience of the Use of Cyanoacrylate Glue in Superficial Venous Disease in an Asian Population: A Pilot Study. ARC Journal of Surgery.2020; 5(4):17-19. DOI: http://dx.doi. org/ 10.20431/2455-572X.0602004.

Copyright: () 2019 Authors. This is an open-access article distributed under the terms of the Creative Commons Attribution License, which permits unrestricted use, distribution, and reproduction in any medium, provided the original author and source are credited. 